\title{
Carboxymethylated chitosan protects rat chondrocytes from NO-induced apoptosis via inhibition of the p38/MAPK signaling pathway
}

\author{
BIN HE, HAIYING TAO, SHIQING LIU, AILIN WEI, FENG PAN, REN CHEN and XIAOHAI LI \\ Department of Orthopaedics, Renmin Hospital of Wuhan University, Wuhan, Hubei 430060, P.R. China
}

Received February 15, 2015; Accepted December 3, 2015

DOI: $10.3892 / \mathrm{mmr} .2016 .4772$

\begin{abstract}
In the present study, the effect of carboxymethylated chitosan (CMCS) on nitric oxide (NO)-induced apoptosis, and activation of the $\mathrm{p} 38 / \mathrm{MAPK}$ signaling pathway in chondrocytes were investigated. Cartilage was isolated from the knee joints of Sprague-Dawley rats, and was used to establish cultured primary chondrocytes. The chondrocytes were incubated with the NO donor, sodium nitroprusside (SNP), to induce apoptosis, and were treated with CMCS and the p38 inhibitor, SB203580. Cell viability was assessed using a Cell Counting Kit-8 assay. Apoptosis of the chondrocytes was detected using Annexin V-fluorescein isothiocyanate/propidium iodide staining. The activation of p38 was detected using Western blot analysis, and caspase- 3 activity was detected using a caspase-3 detection kit. The results indicated that, in chondrocytes treated with SNP, the optical density values of the experimental groups were significantly lower, compared with the control group $(\mathrm{P}<0.05)$. The exposure of the cells to CMCS significantly prevented apoptosis $(\mathrm{P}<0.05)$, and a dose-dependent effect was demonstrated using fluorescence-activated cell sorting analysis $(\mathrm{P}<0.05)$. Examination of the expression and activity of p38 and caspase-3, respectively, showed that SNP increased the expression of p38 and activity of caspase-3, and this trend was reversed following the addition of CMCS and SB203580. Taken together, these findings indicated that CMCS prevented NO-induced apoptosis of chondrocytes via inhibition of the p38/mitogen-activated protein kinase signaling pathway in vitro.
\end{abstract}

\section{Introduction}

Osteoarthritis (OA) is a common, predominantly age-related joint disorder, which is characterized by the loss of

Correspondence to: Dr Bin He, Department of Orthopaedics, Renmin Hospital of Wuhan University, 238 Jiefang Road, Wuchang, Wuhan, Hubei 430060, P.R. China

E-mail: hebin@whu.edu.cn

Key words: osteoarthritis, chondrocyte, apoptosis, carboxymethylated chitosan, nitric oxide chondrocytes, degradation of the extracellular matrix (ECM), subchondral bone remodeling and synovial inflammation (1). It has been demonstrated that chondrocyte apoptosis is associated with the initiation and severity of articular cartilage degradation (2), and chondrocyte apoptosis increases in human OA cartilage (3). Chondrocyte apoptosis is predominantly responsible for the progressive cartilage degradation, which occurs in osteoarthritis (OA) and has gradually become one of the potential therapeutic targets for OA (4).

Mitogen-activated protein kinase (MAPK) is a serine/threonine kinase, the signaling cascades of which control complex programs, including proliferation, differentiation and apoptosis $(5,6)$. There are three major classes of MAPKs in mammals, the extracellular signal-regulated kinases (ERKs), c-jun N-terminal kinase and p38 (7). Chondrocyte apoptosis in experimental OA is regulated by the p38/MAPK signaling pathway (8), and the $\mathrm{p} 38$ /MAPK signal transduction pathway has also been implicated as a critical factor in nitric oxide (NO)-induced articular chondrocyte apoptosis in rabbits (9). The p38/MAPK cascades can be activated or phosphorylated by a series of inflammatory factors and stimuli from the environment, and phosphorylated (p)-p38 is then shuttled into the nucleus, where it activates transcription factors, initiates the expression of upstream apoptosis-associated genes and promotes the apoptosis of chondrocytes $(10,11)$. Caspase- 3 is a key member of the caspase family, which can cleave DNA repair proteins, including poly (ADP-ribose) polymerase, cytoskeletal proteins and inhibitor of caspase-activated deoxytribonuclease, thereby leading to the fragmentation of DNA and eventual cell apoptosis (12). NO is an important physiological and pathological signaling molecule, which can be produced by inflammatory stimuli, including sodium nitroprusside (SNP). It can inhibit the proliferation of chondrocytes and induce chondrocyte apoptosis, thereby damaging cartilage and impairing joint cartilage repair (13). NO can induce chondrocyte apoptosis through multiple pathways, including the p38/MAPK pathway. Inhibiting p38 signaling may effectively inhibit these negative effects, prevent chondrocyte apoptosis and promote cartilage repair.

Carboxymethylated chitosan (CMCS) is a soluble derivative of chitosan, which possesses several desirable physiochemical and biological features. Our previous studies showed that CMCS significantly suppresses the degeneration of cartilage in $\mathrm{OA}$ and protects chondrocytes from interleukin-1 $\beta$-induced 
apoptosis $(14,15)$. It has been found that CMCS can stimulate the proliferation and synthesis of nerve growth factor in Schwann cells (SCs) by activation of the MAPK kinase (MEK)/ERK, phosphatidylinositol 3-kinase/Akt and Wnt/ $\beta$-catenin signaling pathways $(16,17)$, and protects SCs and nucleus pulposus cells from hydrogen peroxide-induced apoptosis $(18,19)$. However, whether CMCS can inhibit chondrocyte apoptosis through inhibiting p38/MAPK signaling remains to be elucidated.

The aim of the present study was to investigate whether CMCS can inhibit chondrocyte apoptosis using an in vitro model of rat articular chondrocyte apoptosis. Numerous studies have indicated that the p38/MAPK signaling pathway is one of the most important signaling pathways in NO-induced cell apoptosis, including chondrocytes (9). Therefore, the present study also aimed to elucidate the mechanism underlying the protective role of CMCS on chondrocytes by examining activation of the p38/MAPK signaling pathway.

\section{Materials and methods}

Animals and reagents. A total of 24 healthy Sprague-Dawley (SD) rats (3 weeks old) with an average body weight of $362 \pm 35 \mathrm{~g}$ were obtained from the Experimental Animal Center of Wuhan University (Wuhan, China). Dulbecco's modified Eagle's medium (DMEM) was obtained from Gibco (Thermo Fisher Scientific, Inc., Waltham, MA, USA), fetal bovine serum (FBS) was obtained from GE Healthcare Life Sciences (Logan, UT, USA). CMCS (purity >99\%) was supplied by the Institute of Chemistry and Environmental Science of Wuhan University (Wuhan, China). Primers were synthesized by Invitrogen (Thermo Fisher Scientific, Inc.). Anti-p38 and anti-p-p38 antibodies were obtained from Cell Signaling Technology, Inc. (Beverly, MA, USA). Anti- $\beta$-actin antibody was obtained from Santa Cruz Biotechnology, Inc. (Santa Cruz, CA, USA). A Caspase-3 Activity Assay kit (cat. no. C1115) was purchased from the Beyotime Institute of Biotechnology (Haimen, China). A Cell Counting Kit-8 (CCK-8; cat. no. CK04) was purchased from Dojindo Molecular Technologies, Inc. (Kumamoto, Japan). SB203580 (cat. no. S3807), type-2 collagenase (cat. no. C6885) and SNP (cat. no. 1614501) were obtained from Sigma-Aldrich (St. Louis, MO, USA). An Annexin V-Fluorescein isothiocyanate (FITC)/propidium iodide (PI) Apoptosis Detection kit (cat. no. 556547) was obtained from BD Biosciences (Franklin Lakes, NJ, USA). Other commonly used reagents were of high purity and commercially available.

Cell isolation and culture. Rat articular chondrocytes were isolated and cultured, as previously described (20). The utilization of rat articular cartilage was approved by the Animal Ethics Committee of Wuhan University. Rats were anesthetized with a single intraperitoneal injection of $1 \%$ sodium pentobarbital $(40 \mathrm{mg} / \mathrm{kg}$; purchased from Sigma-Aldrich; cat. no. 1507002). The articular cartilage tissue was removed from the knee joints of the 3-week-old rats using a scalpel. The extracted cartilage was cut into $1 \times 1 \times 1 \mathrm{~mm}^{3}$ sections. Animals were sacrificed by an overdose of sodium pentobarbital. Subsequently, chondrocytes were isolated from the extracted cartilage by enzymatic digestion with $0.25 \%$ trypsin
(Sigma-Aldrich) in phosphate-buffered saline (PBS) for $1 \mathrm{~h}$, and $0.2 \%$ type- 2 collagenase (381 U/mg; Sigma-Aldrich) in DMEM for $4 \mathrm{~h}$. Following collection of individual cells by brief centrifugation, the chondrocytes were resuspended in DMEM supplemented with 10\% FBS, 100 U/ml streptomycin and $100 \mathrm{U} / \mathrm{ml}$ penicillin (Hyclone, Logan, UT, USA). The culture medium was replaced every other day, and the second and third passage of chondrocytes were used in the subsequent experiments.

Cell treatment and grouping. To establish the apoptotic model of cultured chondrocytes, SNP was used, which generates NO (21). Briefly, the chondrocytes $\left(5 \times 10^{4}\right.$ cells $\left./ \mathrm{cm}^{2}\right)$ were seeded and cultured at $37^{\circ} \mathrm{C}$ for $24 \mathrm{~h}$, following which the cells were randomly divided into five groups, in which the medium was replaced with complete medium (DMEM with $10 \%$ FBS and antibiotics) containing $0,0.5,1,2$ or $3 \mathrm{mM} \mathrm{SNP}$, cultured at $37^{\circ} \mathrm{C}$ for different durations (3, 6, 12 and $\left.24 \mathrm{~h}\right)$. CMCS was added into the culture medium at different concentrations (50, 100 and $200 \mu \mathrm{g} / \mathrm{ml}$ ). Subsequently, in order to determine the activation of the p38/MAPK signaling pathway, the p38/MAPK specific inhibitor, SB203580 was used (22).

Cell viability assay. The chondrocytes were cultured in 96-well microplates at a density of $2 \times 10^{4}$ cells per well with DMEM containing $0.1 \% \mathrm{FBS}$ at $37^{\circ} \mathrm{C}$ for $24 \mathrm{~h}$, and then exposed to different concentrations of SNP $(0.5,1,2$ and $3 \mathrm{mM}), \mathrm{CMCS}$ $(50,100$ and $200 \mu \mathrm{g} / \mathrm{ml})$ or SB203580 $(10 \mu \mathrm{M})$ for $24 \mathrm{~h}$, and for different durations $(0,3,6,12$ or $24 \mathrm{~h})$. For the quantitative analysis of the cell viability, $10 \mu \mathrm{lCCK}-8$ solution was added to each well and incubated at $37^{\circ} \mathrm{C}$ for $1 \mathrm{~h}$. The optical densities were measured at $450 \mathrm{~nm}$ using an ELx800 Absorbance Microplate reader (BioTek Instruments, Inc., Winooski, VT, USA). Cell viability was determined as a percentage of the number of control (untreated) cells. Viability in the control group was designated as $100 \%$, and cell viability in the treatment groups was determined as follows:

Viability $(\%$ of control $)=[(\mathrm{Ae}-\mathrm{Ab}) /(\mathrm{Ac}-\mathrm{Ab})] \times 100 \%$. Ae, $\mathrm{Ab}$ and $\mathrm{Ac}$ represent the A450 of the experimental, blank and control groups, respectively. All experiments were performed in triplicate in three independent experiments.

Flow cytometric analysis. The apoptotic rates of the chondrocytes was measured using flow cytometry (FCM), according to the manufacturer's protocol of the Annexin V-FITC/PI Apoptosis Detection kit. In brief, the chondrocytes were treated with SNP $(0.5,1,2$ and $3 \mathrm{mM}), \operatorname{CMCS}(50,100$ and $200 \mu \mathrm{g} / \mathrm{ml})$ or SB203580 $(10 \mu \mathrm{M})$. Following culture of the chondrocytes for the indicated durations $(0,3,6,12$ or $24 \mathrm{~h})$, trypsinization was performed with $0.25 \%$ trypsin without ethylene diamine tetraacetic acid for analysis of apoptosis using the Annexin V-FITC/PI Apoptosis Detection kit. Cell apoptosis was analyzed using a BD FACSVerse ${ }^{\mathrm{TM}}$ flow cytometer (Becton Dickinson, Heidelberg, Germany) at $488 \mathrm{~nm}$. The data were analyzed using CellQuest ${ }^{\mathrm{TM}}$ software (version 4.01; Becton Dickinson).

Caspase-3 activity assay. Caspase-3 activity was determined using a colorimetric assay kit (cat.no. C1115; Beyotime Institute of Biotechnology). The chondrocytes were first cultured in 
a mixture of DMEM supplemented with 5\% FBS, SNP and CMCS. Following incubation at $37^{\circ} \mathrm{C}$ for $24 \mathrm{~h}$, the cells were treated, according to the manufacturer's protocol. The cell lysates were prepared, and assays were performed in 96-well plates (at a density of $2 \times 10^{4}$ cells/well) by incubating $10 \mu \mathrm{l}$ cell lysate per sample in $80 \mu \mathrm{l}$ reaction buffer $(1 \% \mathrm{NP}-40$, $20 \mathrm{mM}$ Tris- $\mathrm{HCl}, 137 \mathrm{mM}$ NAD and $10 \%$ glycerol) containing $10 \mu \mathrm{l}$ caspase-3 substrate (Ac-DEVD-pNA; Beyotime Institute of Biotechnology). The cell lysates were incubated at $37^{\circ} \mathrm{C}$ for $4 \mathrm{~h}$. The absorbance at $405 \mathrm{~nm}$ was read using an ELx800 Absorbance Microplate reader (BioTek Instruments, Inc.). Caspase-3 activities were determined as the percentage of enzyme activity, compared with the control. All experiments were performed in triplicate.

Reverse transcription-quantitative polymerase chain reaction $(R T-q P C R)$ analysis. The mRNA of the treated chondrocytes was extracted using TRIzol reagent (Invitrogen; Thermo Fisher Scientific, Inc.), according to the manufacturer's protocol. An ABI Prism 7500 real-time PCR system (Applied Biosystems; Thermo Fisher Scientific, Inc.) was used to detect the mRNA expression levels of the target genes. The sequences of the primers used, for B cell lymphoma (Bcl)-2, Bcl-2-associated X protein (Bax) and GAPDH, are shown in Table I.

For RT reaction, $5 \mu \mathrm{lmRNA}$ was reverse transcribed into cDNA and amplified using specific reverse primers of the target genes using a RevertAid ${ }^{\mathrm{TM}}$ First Strand cDNA Synthesis kit (Fermentas; Thermo Fisher Scientific, Inc.). The PCR reaction mixture included $1 \mu 1$ forward and reverse primers $(10 \mu \mathrm{M}), 1 \mu \mathrm{l}$ SYBR Green I fluorescent dye and $1 \mu \mathrm{l}$ cDNA. The PCR conditions were as follows: Initial step at $95^{\circ} \mathrm{C}$ for $2 \mathrm{~min}$, followed by 40 cycles at $95^{\circ} \mathrm{C}$ for $20 \mathrm{sec}$ and $60^{\circ} \mathrm{C}$ for $40 \mathrm{sec}$. The relative expression levels of target genes were analyzed using the comparative cycle threshold method (23). Data were standardized by GAPDH. The results were obtained from three independent experiments.

Western blot analysis. The chondrocytes were treated with SNP, with or without CMCS, following which the chondrocytes were lysed in lysis buffer at $4^{\circ} \mathrm{C}$ for $30 \mathrm{~min}$. The lysates were centrifuged at $13,000 \mathrm{~g}$ or $15 \mathrm{~min}$ at $4^{\circ} \mathrm{C}$. The supernatants were collected and stored at $-80^{\circ} \mathrm{C}$. Protein concentrations were determined using a bicinchoninic acid method (Pierce Biotechnology; Thermo Fisher Scientific, Inc.). Total protein $(50 \mu \mathrm{g})$ was separated by $12 \%$ sodium dodecyl sulfate-polyacrylamide gel electrophoresis (Beyotime Institute of Biotechnology) and electroblotted onto polyvinylidene difluoride membrane (EMD Millipore, Billerica, MA, USA). After being blocked with $5 \%$ non-fat milk in Tris-buffered saline, the membrane was incubated overnight at $4^{\circ} \mathrm{C}$ with primary antibodies against p38 (rabbit polyclonal IgG, cat. no.9212; Cell Signaling Technology, Inc.), p-p38 (rabbit polyclonal IgG, cat. no. 9211; Cell Signaling Technology, Inc.) and $\beta$-actin (rabbit polyclonal IgG, cat. no. sc-10731; Santa Cruz Biotechnology, Inc.) at dilutions of 1:200 for $2 \mathrm{~h}$ at room temperature. Subsequently, the membrane was incubated with a peroxidase-conjugated secondary antibody (goat anti-rabbit horseradish peroxidase-conjugated polyclonal $\mathrm{IgG}$, cat. no. sc-45101; Santa Cruz Biotechnology, Inc.) for $1 \mathrm{~h}$ at
Table I. Primer sequences of target genes.

\begin{tabular}{lcc}
\hline Gene & Primer sequence & $\begin{array}{c}\text { Product } \\
\text { size (bp) }\end{array}$ \\
\hline Bcl-2 & F: 5'-GCGTCAACAGGGAGATGTCA-3' & 225 \\
& R: 5'-GGTATGCACCCAGAGTGATG-3' & \\
Bax & F: 5'-GGCGAATTGGAGATGAACTG-3' & 209 \\
& R: 5'-GATCAGCTCGGGCACTTTAG-3' & \\
GAPDH & F: 5'-TGTCTCCTGCGACTTCAACAG-3' & 256 \\
& R: 5'-GAGGCCATGTAGGCCATGAG-3' &
\end{tabular}

Bcl-2, B cell lymphoma-2; Bax, Bcl-2-associated X protein; F, forward; $R$, reverse.

room temperature. Signals were detected by enhanced chemiluminescence. Optical band density was quantified using a Geliance 200 Imaging system (PerkinElmer, Waltham, MA, USA) and Gene Snap software (version 6.08.04; Syngene, Cambridge, UK). The values for p38 and p-p38 were normalized to that of $\beta$-actin.

Statistical analysis. The data are presented as the mean \pm standard deviation. Statistical significance was analyzed by one-way analysis of variance using SPSS, version 16.0 (SPSS, Inc., Chicago, IL, USA). $\mathrm{P}<0.05$ was considered to indicate a statistically significant difference.

\section{Results}

CMCS increases cell viability in SNP-induced chondrocytes. To determine the protective effects of CMCS on chondrocytes, a model of apoptosis was established in the present study by exposure of chondrocytes to SNP, an inorganic compound with the formula $\mathrm{Na}_{2}\left[\mathrm{Fe}(\mathrm{CN})_{5}-\mathrm{NO}\right] \cdot 2 \mathrm{H}_{2} \mathrm{O}$. As shown in Fig. 1A, SNP induced a dose-dependent cytotoxic effect on the chondrocytes, and treatment with $3 \mathrm{mM}$ SNP for $24 \mathrm{~h}$ induced a decrease in cell viability by $>90 \%$. In addition, treatment with $3 \mathrm{mM}$ SNP for different durations induced a time-dependent cytotoxic effect (Fig. 1B). Therefore, the results indicated that SNP induced dose- and time-dependent cytotoxic effects on the chondrocytes. Based on these data, chondrocytes treated with $3 \mathrm{mM}$ SNP for $24 \mathrm{~h}$ were used as the in vitro apoptosis model for subsequent experiments. To investigate the protective effects of CMCS on chondrocytes, the chondrocytes were pretreated with different concentrations of CMCS for $24 \mathrm{~h}$ prior to SNP induction, following which cell viability was measured. As shown in Fig. 1C, CMCS caused an increase in SNP-induced cytotoxicity, indicating that CMCS protected the chondrocytes from the SNP-induced decrease in viability, and may offer potential as a potent drug to treat OA.

CMCS inhibits SNP-induced apoptosis in rat chondrocytes. To confirm the apoptosis-inducing effects of SNP, Annexin V-FITC/PI staining, followed by FCM was performed. As shown in Fig. 2, the numbers of normal cells in the SNP-induced groups were significantly lower, compared with the number in the control group, whereas the numbers of early apoptotic, 
A

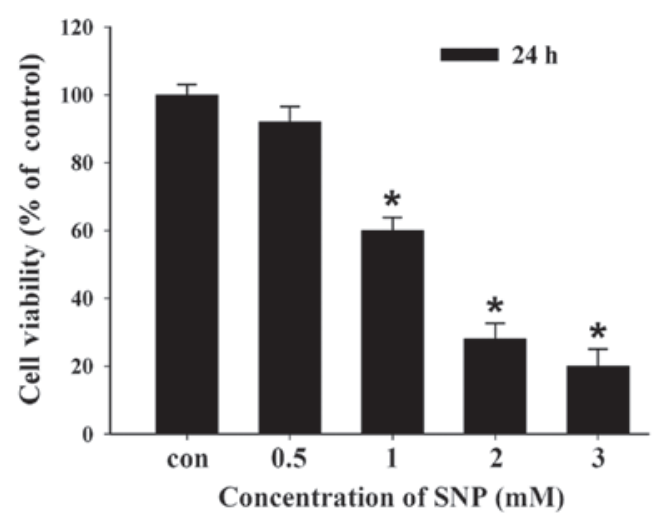

B

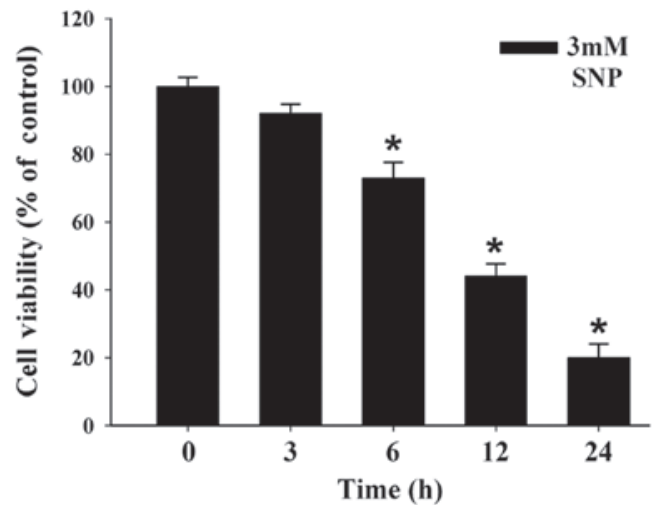

C



Figure 1. Protective effects of CMCS on SNP-induced cytotoxicity of chondrocytes. (A) Cell viability of chondrocytes treated with different concentrations of SNP for $24 \mathrm{~h}$. (B) Cell viability of chondrocytes treated with $3 \mathrm{mM}$ SNP for different durations. (C) Chondrocytes were pretreated with different concentrations $(1,50,100$ and $200 \mu \mathrm{g} / \mathrm{ml})$ of CMCS for $24 \mathrm{~h}$, and were then treated with $3 \mathrm{mM}$ of SNP for $12 \mathrm{~h}$. Cell viability was then determined using a Cell Counting Kit- 8 assay. Data are presented as the mean \pm standard deviation. ${ }^{*} \mathrm{P}<0.05$, vs. con; ${ }^{~} \mathrm{P}<0.05$, vs. $3 \mathrm{mM}$ SNP-treated group. CMCS, carboxymethylated chitosan; SNP, sodium nitroprusside; con, control.

late apoptotic and dead cells were significantly higher in number, compared with those in the control group. Increasing concentrations and durations increased the apoptotic rate, and the maximum pro-apoptotic effect was observed following treatment with $3 \mathrm{mM}$ SNP for $24 \mathrm{~h}$. These results are consistent with previous studies, demonstrating that SNP treatment leads to chondrocyte apoptosis $(24,25)$. Following treatment with different concentrations of CMCS, the proportion of early apoptotic, late apoptotic and dead cells decreased, and there was a dose-dependent effect of CMCS treatment, with $200 \mu \mathrm{g} / \mathrm{ml} \mathrm{CMCS}$ exerting the most marked inhibitory effect on SNP-induced apoptosis.
CMCS inhibits the activation of p38 and decreases caspase-3 activity. To further examine the mechanism underlying the effects of CMCS on signaling cascades, the present study analyzed the activation of p38/MAPK. As shown in Fig. 3, quantification of the Western blot bands showed that the protein levels of p-p38 were significantly higher in the SNP-treated groups, compared with the control group. Following treatment with CMCS or the p38 inhibitor, SB203580, the expression of p-p38 was decreased. As shown in Fig. 4, caspase-3 activity was significantly increased, to $63 \%$ of the control, in the SNP-induced chondrocytes, however, pretreatment with 50, 100 or $200 \mu \mathrm{g} / \mathrm{ml} \mathrm{CMCS}$ attenuated the SNP-induced increase in caspase- 3 activity by $\sim 41,54$ and $56 \%$, respectively.

CMCS increases the expression of Bcl-2 and decreases the expression of Bax in SNP-induced chondrocytes. In order to further investigate the mechanisms underlying the effects of CMCS on SNP-induced chondrocyte apoptosis, the mRNA expression levels of pro-apoptotic Bax and anti-apoptotic Bcl-2 were detected using RT-qPCR analysis. As shown in Fig. 5A, the mRNA expression levels of Bcl-2 were markedly elevated in the CMCS-treated chondrocytes, compared with the $3 \mathrm{mM}$ SNP-treated group without CMCS, at CMCS concentrations of $50 \mu \mathrm{g} / \mathrm{ml}(1.68 \pm 0.16$-fold), $100 \mu \mathrm{g} / \mathrm{ml}$ (2.23 \pm 0.21 -fold) and $200 \mu \mathrm{g} / \mathrm{ml}(4.12 \pm 0.23$-fold). As shown in Fig. 5B, $3 \mathrm{mM}$ SNP significantly increased the mRNA level of Bax (2.14 \pm 0.17 -fold), compared with the control group. This increase was reversed by the addition of CMCS. These findings indicated that CMCS inhibited the mRNA expression of Bax and promoted the mRNA expression of $\mathrm{Bcl}-2$ in the SNP-induced chondrocytes.

CMCS prevents SNP-induced apoptosis via p38/MAPK. As p38/MAPK was found to be activated in response to SNP., the present study proceeded to address the role of p38/MAPK in the CMCS-induced inhibition of chondrocyte apoptosis, by treating the chondrocytes with SB203580, a specific inhibitor of p38 activation. The chondrocytes were pretreated with $10 \mu \mathrm{M}$ SB203580 for $1 \mathrm{~h}$ and then stimulated with CMCS for $24 \mathrm{~h}$. A subsequent CCK-8 assay showed that pretreatment with CMCS and/or SB203580 decreased the inhibitory effect of SNP on viability (Fig. 6A). In addition, FCM showed that pretreatment with CMCS or SB203580 prevented SNP-induced apoptosis (Fig. 6B). These findings suggested that CMCS prevented NO-induced apoptosis, possibly through the inhibition of p38/MAPK activation.

\section{Discussion}

CMCS has shown therapeutic effects on non-alcoholic fatty liver disease and hydrophobic drug delivery $(26,27)$. Our previous study indicated that CMCS inhibits chondrocyte, $\mathrm{SC}$ and nucleus pulposus cell apoptosis in vitro $(15,18,19)$. However, the mechanism or signaling pathway underlying the effect of CMCS in OA remains to be fully elucidated. The results of the present study indicated that pre-treatment with CMCS in SNP-induced chondrocytes promoted the expression of Bcl-2, inhibited the expression of Bax and reduced caspase-3 activation. These effects by CMCS are partially mediated via the inhibition of p38/MAPK signaling. 
A

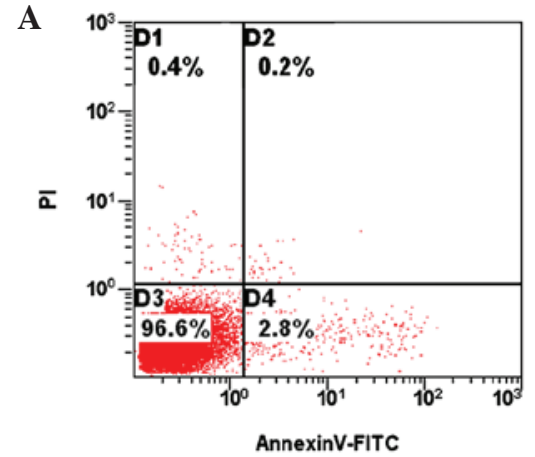

D

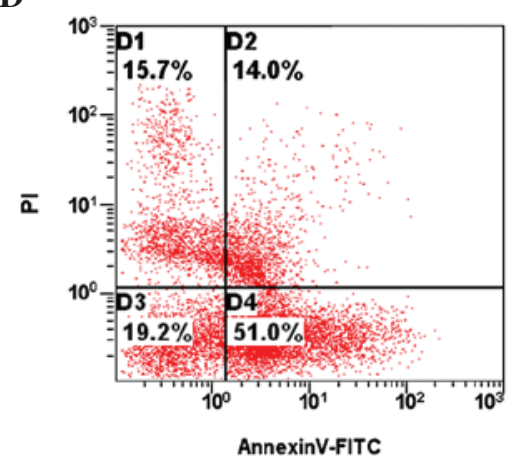

B

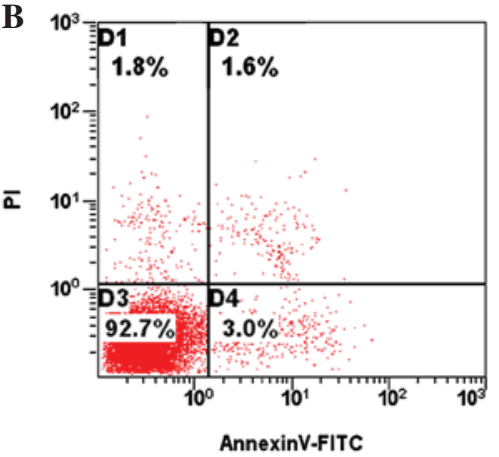

$\mathbf{E}$

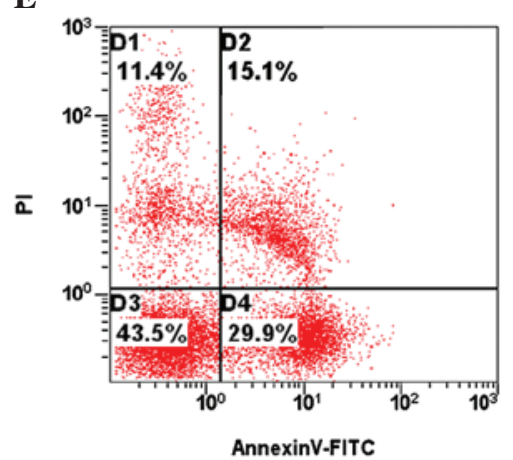

C



$\mathbf{F}$



Figure 2. Effect of CMCS on apoptosis in SNP-induced chondrocytes. (A) Control group (phosphate-buffered saline). (B) $200 \mu \mathrm{g} / \mathrm{ml} \mathrm{CMCS-treated} \mathrm{group}$ (C) $3 \mathrm{mM}$ SNP-induced group. (D) $3 \mathrm{mM} \mathrm{SNP}+50 \mu \mathrm{g} / \mathrm{ml}$ CMCS-treated group. (E) $3 \mathrm{mM} \mathrm{SNP}+100 \mu \mathrm{g} / \mathrm{ml} \mathrm{CMCS}$-treated group. (F) $3 \mathrm{mM} \mathrm{SNP}+200 \mu \mathrm{g} / \mathrm{ml}$ CMCS-treated group. The percentages shown in quadrants D1-D4 for each panel represent the following: D1: Annexin V-FITC/PI (-/+), necrotic cells; D2: Annexin V-FITC/PI (+/+), late apoptotic/necrotic cells; D3: Annexin V-FITC/PI (-/-), live cells; and D4: Annexin V-FITC/PI (+/-): early apoptotic cells. CMCS, carboxymethylated chitosan; SNP sodium nitroprusside; FITC, fluorescein isothiocyanate PI, propidium iodide.

A

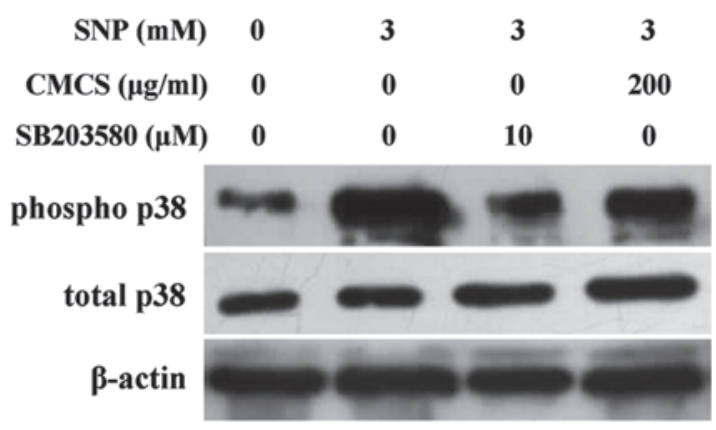

B

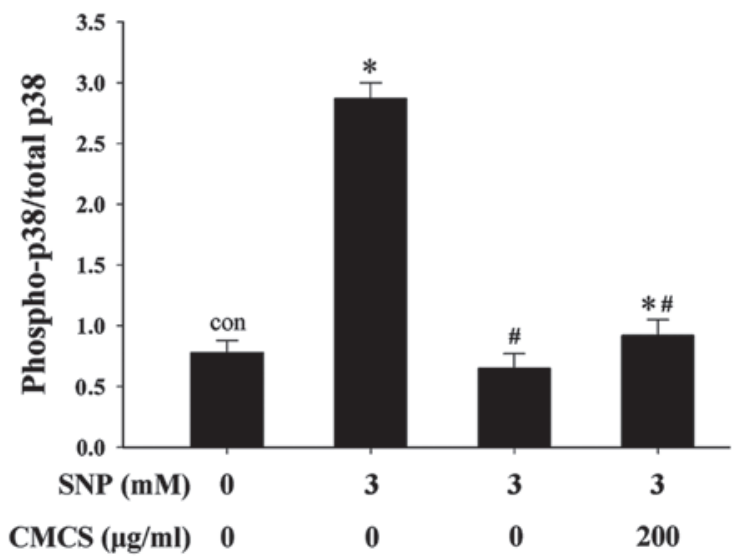

Figure 3. Effect of CMCS on the activation of p38. (A) Western blot analysis was performed to detect the protein expression levels of p38 and phospho-p38 following SNP-induced apoptosis, with without CMCS or SB203580 pretreatment. (B) Densitometric analysis of the representative Western blots. Data were normalized to the expression of $\beta$-actin and presented as a percentage of the untreated control. ${ }^{*} \mathrm{P}<0.05$, vs. con; ${ }^{\#} \mathrm{P}<0.05$, vs. $3 \mathrm{mM} \mathrm{SNP}$-induced group. $\mathrm{CMCS}$, carboxymethylated chitosan; SNP sodium nitroprusside; phospho, phosphorylated; con, control.

OA is a degenerative joint disease with multiple underlying pathogenic mechanisms, caused by various risk factors, including NO (28), polychlorinated biphenyl 126 (29), bupivacaine and levobupivacaine (30), and C/EBP homologous protein (31) has been reported to induce apoptosis in chondrocytes. As these apoptosis-inducing substances may be involved in the pathogenesis of OA, manipulation of the mechanism mediated by these stimuli has the potential for substantial therapeutic effects.

The loss of chondrocyte function, ECM degradation and apoptosis are crucial in the progression of OA, thus inhibiting the development of these factors is important for the treatment of OA. To the best of our knowledge, the signaling pathways involved in the effects of CMCS on OA remain to be fully elucidated. In the present study, the anti-apoptotic effect of CMCS was evaluated using a classical apoptosis model induced by NO.

Chondrocyte apoptosis is key in the degeneration and degradation of articular cartilage in cases of OA. Reduced cellularity is a characteristic feature of OA cartilage, and apoptosis has been suggested as an underlying cause (32).

In the present study, the results of a CCK- 8 assay revealed that cell viability in the articular chondrocytes following 


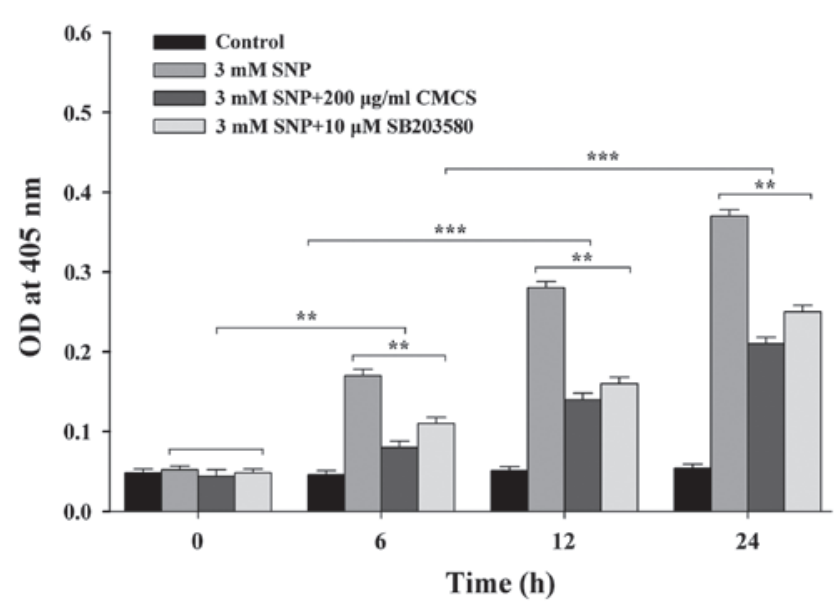

Figure 4. Effect of CMCS on caspase-3 activity in SNP-induced chondrocytes. ${ }^{* *} \mathrm{P}<0.05$ vs. other groups at same time intervals; ${ }^{* * *} \mathrm{P}<0.05$, vs. different time intervals. CMCS, carboxymethylated chitosan; SNP sodium nitroprusside; OD, optical density.

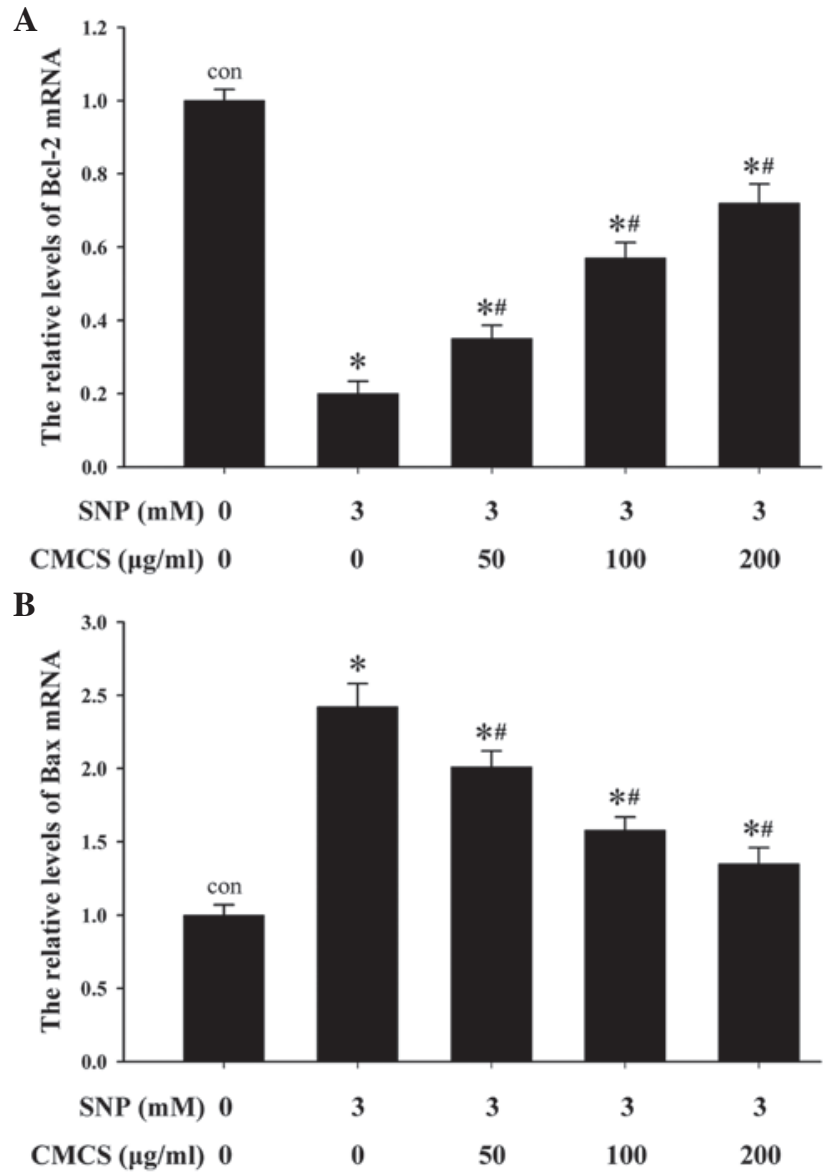

Figure 5. mRNA expression levels of Bcl-2 and Bax in different groups. mRNA expression levels of (A) Bcl-2 and (B) Bax were detected using reverse transcription-quantitative polymerase chain reaction analysis. ${ }^{\text {*P }}<0.05$, vs. con; ${ }^{\prime} \mathrm{P}<0.05$, vs. $3 \mathrm{mM}$ SNP-treated group. CMCS, carboxymethylated chitosan; SNP sodium nitroprusside; Bcl-2, B-cell lymphoma-2; Bax, Bcl-2-associated X protein; con, control.

SNP induction were markedly decreased, compared with those in normal chondrocytes, and cell viability correlated positively with the percentage of apoptotic chondrocytes. This suggested that reduced cellularity in OA cartilage may, at least
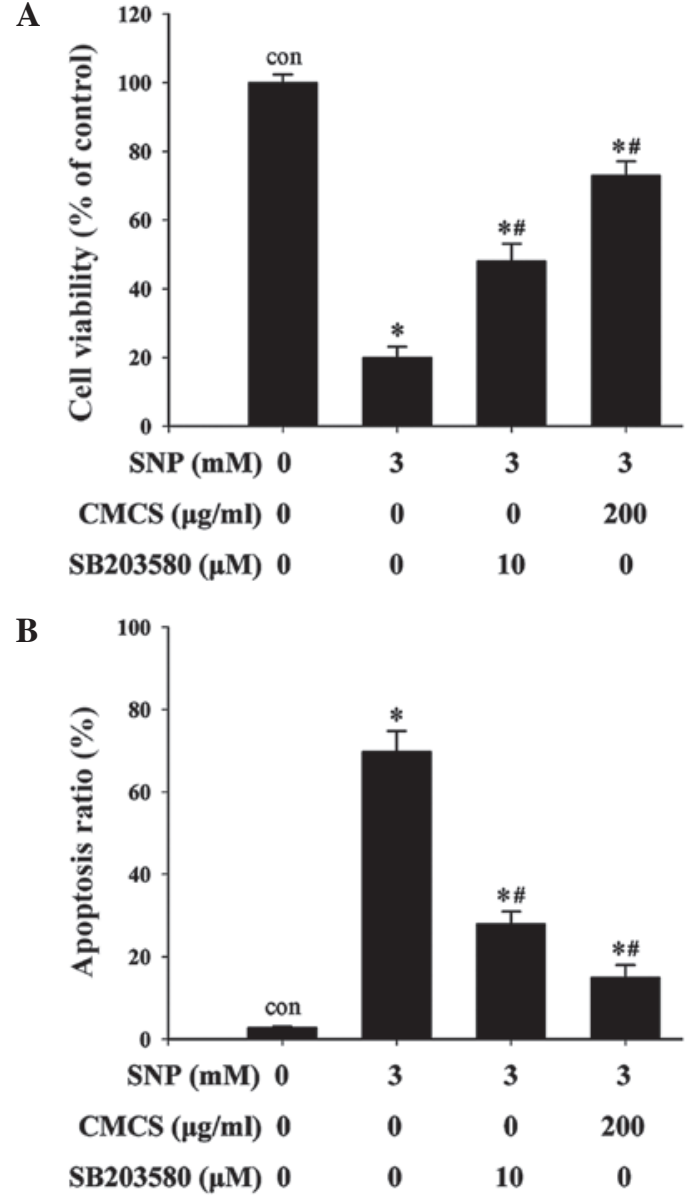

Figure 6. Effect of SB203580 or CMCS on proliferation and apoptosis in SNP-induced chondrocytes. (A) Proliferation of chondrocytes was detected using a Cell Counting Kit-8. (B) Apoptosis was analyzed by flow cytometry. ${ }^{\text {"P }}<0.05$, vs. con; ${ }^{\text {"P}} \mathrm{P}<0.05$, vs. $3 \mathrm{mM}$ SNP-treated group. CMCS, carboxymethylated chitosan; SNP sodium nitroprusside; con, control.

partially, be attributed to cell death by apoptosis. The results from the FCM showed that CMCS suppressed apoptosis of the SNP-induced chondrocytes. These results were confirmed in the in vitro experiments in the present study, which revealed that CMCS inhibited the increase in the proportion of early and late apoptotic cells in the NO-induced chondrocytes, suggesting that CMCS prevented the degeneration and degradation of articular cartilage by inhibiting chondrocyte apoptosis.

Apoptosis is an autonomous process of programmed cell death, regulated by multiple signaling pathways. One of the important pathways during the apoptotic process involves the activation of caspase- 3 , and induction of the hydrolysis of nucleic acids and cytoskeletal proteins (33). In vitro studies have confirmed that caspase- 3 activity is increased significantly in NO-induced chondrocyte apoptosis $(34,35)$. Notably, caspase- 3 is activated by cytochrome $c$ (Cyt $c$ ), which is induced by apoptotic signals. These signaling events are set in motion by the pro-apoptotic protein, Bax, a member of the Bcl-2 family, which migrates to the mitochondrial membrane and induces the release of Cyt $c(36,37)$. Caspase- 3 is a member of the downstream Cyt $c$ signaling pathway, and is the most important executor of cell apoptosis. A previous study reported that millimeter wave treatment inhibited p38/MAPK signaling and inhibited caspase- 3 activation and chondrocyte 
apoptosis (20). Therefore, inhibiting the activation of caspase-3 may be the key to decreasing chondrocyte apoptosis. The results of the present study indicated that CMCS inhibited the activation of caspase- 3 induced by NO, and this may be one of the most important anti-apoptotic mechanisms for CMCS.

The present study demonstrated that CMCS treatment inhibited NO-induced effects on chondrocytes in vitro, including apoptosis and the expression of caspase-3, and effects involving inhibition of the p38/MAPK signaling pathway. CMCS $(50,100$ and $200 \mu \mathrm{g} / \mathrm{ml})$ protected chondrocytes from SNP-induced apoptosis, and inhibited the apoptotic rates of NO-induced rat chondrocytes, with effects on the expression levels of Bcl-2 and Bax. CMCS also reduced caspase-3 activity in the SNP-induced chondrocytes, and suppressed p38 phosphorylation, suggesting that the p38/MAPK signaling pathway may represent a target for the effects of CMCS, which may offer potential in the treatment of OA.

There were several limitations to the present study. Notably, all experiments were performed in a cell-based in vitro system, and whether equivalent effects are be observed consistently in an in vivo model and in patients remains to be elucidated. Furthermore, all data were acquired with CMCS-pretreated cells, which appeared to be essential for the protective effects of the compound in the present study and in previous reports (15-19). Further investigations, to examine whether the pre-treated cells have altered signaling pathways in SNP and other biological process, may assist in improving the preparation of cells against apoptotic treatment.

In conclusion, the present study demonstrated that CMCS inhibited SNP-induced chondrocyte apoptosis, promoted the expression of Bcl-2, and inhibited the expression of Bax and caspase-3 activity via its effects on the p38/MAPK signaling pathway. Collectively, these results indicated that CMCS may have a potential therapeutic role for the treatment of OA.

\section{Acknowledgements}

This study was supported by the National Natural Science Foundation of China (grant no. 81301056) and the Natural Science Foundation of Hubei Province (grant no. 2013CKB002).

\section{References}

1. Loeser RF: Aging and osteoarthritis: The role of chondrocytes senescence and aging changes in the cartilage matrix. Osteoarthritis Cartilage 17: 971-979, 2009.

2. Thomas CM, Fuller CJ, Whittles CE and Sharif M: Chondrocyte death by apoptosis is associated with the initiation and severity of articular cartilage degradation. In J Rheum Dis 14: 191-198, 2011.

3. Chen Q, Zhang B, Yi T and Xia C: Increased apoptosis in human knee osteoarthritis cartilage related to the expression of protein kinase B and protein kinase C\&\#945; in chondrocytes. Folia Histochem Cytobiol 50: 137-143, 2012.

4. Huang JG, Xia C, Zheng XP, Yi TT, Wang XY, Song G and Zhang B: $17 \beta$-Estradiol promotes cell proliferation in rat osteoarthritis model chondrocytes via PI3K/Akt pathway. Cell Mol Biol Lett 16: 564-575, 2011.

5. Andjelkov N, Elvenes $\mathrm{J}$, Knutsen $\mathrm{G}$ and Johansen O: Beta-endorphin regulation of MAPKs in cultures human articular chondrocytes: MAPK inhibitors prevent the increase of IL-1 beta protein levels during beta-endorphin stimulation. Cell Commun Adhes 14: 1-8, 2007.

6. Ding L, Guo D and Homandberg GA: The cartilage chondrolytic mechanism of fibronectin fragments involves MAP kanases: Comparison of three fragments and native fibronectin. Osteoarthritis Cartilage 16: 1253-1262, 2008.
7. Kong D, Zheng T, Zhang M, Wang D, Du S, Li X, Fang J and Cao X: Static mechanical stress induces apoptosis in rat endplate chondrocytes through MAPK and mitochondria-dependent caspase activation signaling pathways. PLoS One 8: e69403, 2013.

8. Takebe K, Nishiyama T, Hayashi S, Hashimoto S, Fujishiro T, Kanzaki N, Kawakita K, Iwasa K, Kuroda R and Kurosaka M: Regulation of p38 MAPK phosphorylation inhibits chondrocyte apoptosis in response to heat stress or mechanical stress. Int J Mol Med 27: 329-335, 2011.

9. Wang $\mathrm{H}$, Wang Z, Chen J and Wu J: Apoptosis induced by NO via phosphorylation of p38 MAPK that stimulates NF-kappa B, p53 and caspase- 3 activation in rabbit articular chondrocytes. Cell Biol Int 31: 1027-1035, 2007.

10. Hamamura K, Goldring MB and Yokota H: Involvement of p38 MAPK in regulation of MMP-13 mRNA in chondrocytes in response to surviving stress to endoplasmic reticulum. Arch Oral Biol 54: 279-286, 2009.

11. Namdari S, Wei L, Moore D and Chen Q: Reduced limb length and worsened osteoarthritis in adult mice after genetic inhibition of p38 MAP kinase activity in cartilage. Arthritis Rheum 58: 3520-3529, 2008.

12. Sena P, Manfredini G, Benincasa M, Mariani F, Smargiassi A, Catani F and Palumbo C: Up-regulation of the chemo-attractive receptor ChemR23 and occurrence of apoptosis in human chondrocytesisolated from fractured calcaneal osteochondral fragments. J Anat 224: 659-668, 2014.

13. Chen Q, Gao Y, Kao X, Chen J, Xue W, Xiong Y and Wang Z: SNP-induced apoptosis may be mediated with caspase inhibitor by JNK signaling pathways in rabbit articular chondrocytes. J Toxicol Sci 37: 157-167, 2012.

14. Liu SQ, Qiu B, Chen LY, Peng H and Du YM: The effects of carboxymethylated chitosan on metalloproteinase-1,-3 and tissue inhibitor of metalloproteinase- 1 gene expression in cartilage of experimental osteoarthritis. Rheumatol Int 26: 52-57, 2005.

15. Chen Q, Liu SQ, Du YM, Peng $H$ and Sun LP: Carboxymethyl-chitosan protects rabbit chondrocytes from interleukin-1beta-induced apoptosis. Eur J Pharmacol 541: 1-8, 2006.

16. He B, Liu SQ, Chen Q, Li HH, Ding WJ and Deng M: Carboxymethylated chitosan stimulates proliferation of Schwann cells in vitro via the activation of the ERK and Akt signaling pathways. Eur J Pharmacol 667: 195-201, 2011.

17. Tao HY, He B, Liu SQ, Wei AL, Tao FH, Tao HL, Deng WX, $\mathrm{Li} \mathrm{HH}$ and Chen Q: Effect of carboxymethylated chitosan on the biosynthesis of NGF and activation of the Wnt/ $\beta$-catenin signaling pathway in the proliferation of Schwann cells. Eur J Pharmacol 702: 85-92, 2013.

18. He B, Tao HY and Liu SQ: Neuroprotective effects of carboxymethylated chitosan on hydrogen peroxide induced apoptosis in Schwann cells. Eur J Pharmacol 740: 127-134, 2014.

19. He B, Tao H, Liu S and Wei A: Protective effect of carboxymethylated chitosan on hydrogen peroxide-induced apoptosis in nucleus pulposus cells. Mol Med Rep 11: 1629-1638, 2015.

20. Li X, Du M, Liu X, Wu M, Ye H, Lin J, Chen W and Wu G: Millimeter wave treatment inhibits NO-induced apoptosis of chondrocytes through the p38MAPK pathway. Int J Mol Med 25: 393-399, 2010

21. Liang Q, Wang XP and Chen TS: Resveratrol protects rabbit articular chondrocyte against sodium nitroprusside-induced apoptosis via scavenging ROS. Apoptosis 19: 1354-1363, 2014.

22. Cuenda A, Rouse J, Doza YN, Meier R, Cohen P, Gallagher TF, Young PR and Lee JC: SB203580 is a specific inhibitor of a MAP kinase homologue which is stimulated by cellular stresses and interleukin-1. FEBS Lett 364: 229-233, 1995.

23. Glynn RW, Miller N, Mahon S and Kerin MJ: Expression levels of HER2/neu and those of collocated genes at 17q12-21, in breast cancer. Oncol Rep 28: 365-369, 2012.

24. Kühn K and Lotz M: Mechanisms of sodium nitroprusside-induced death in human chondrocytes. Rheumatol Int 23: 241-247, 2003.

25. Chen Q, Mei X, Han G, Ling P, Guo B, Guo Y, Shao H, Wang G, Cui Z, Bai Y and Xu F: Xanthan gum protects rabbit articular chondrocytes against sodium nitroprusside-induced apoptosis in vitro. Carbohydr Polym 131: 363-369, 2015.

26. Liu X, Yang F, Song T, Zeng A, Wang Q, Sun Z and Shen J: Synthesis of carboxymethylated and quaternized chitosan and their therapeutic effect on nonalcoholic Fatty liver disease. J Agric Food Chem 59: 10683-10692, 2011. 
27. Nam JP,Park SC, Kim TH, Jang JY, Choi C, Jang MK and Nah JW: Encapsulation of paclitaxel into lauric acid-O-carboxymetlyl chitosan transferring micelles for hydrophobic drug delivery and site-specific targeted delivery. Int J Pharm 457: 124-135, 2013.

28. Blanco FJ, Ochs RL, Schwarz H and Lotz M: Chondrocyte apoptosis induced by nitric oxide. Am J Pathol 146: 75-85, 1995.

29. Lee HG and Yang JH: PCB126 induces apoptosis of chondrocytes via ROS-dependent pathways. Osteoarthritis Cartilage 20: $1179-1185,2012$.

30. Gungor I, Yilmaz A, Ozturk AM, Ergun MA, Menevse S and Kaya K: Bupivacaine and levobupivacaine induce apoptosis in rat chondrocyte cell cultures at ultra-low doses. Eur J Orthop Surg Traumatol 24: 291-295, 2014.

31. Uehara Y, Hirose J, Yamabe S, Okamoto N, Okada T, Oyadomari S and Mizuta $\mathrm{H}$ : Endoplasmic reticulum stress-induced apoptosis contributes to articular cartilage degeneration via C/EBP homologous protein. Osteoarthritis Cartilage 22: 1007-1017, 2014.
32. Hwang HS and Kim HA: Chondrocyte apoptosis in the pathogenesis of osteoarthritis. Int J Mol Sci 16: 26035-26054, 2015.

33. Li J and Yuan J: Caspases in apoptosis and beyond. Oncogene 27 6194-6206, 2008.

34. Wang H, Wang Z, Chen J and Wu J: Apoptosis induced by NO via phosphorylation of p38 MAPK that stimulates NF-kappaB, p53 and caspase- 3 activation in rabbit articular chondrocytes. Cell Biol Int 31: 1027-1035, 2007.

35. Sakata S, Hayashi S, Fujishiro T, Kawakita K, Kanzaki N, Hashimoto S, Iwasa K, Chinzei N, Kihara S, Haneda M, et al: Oxidative stress-induced apoptosis and matrix loss of chondrocytes is inhibited by eicosapentaenoic acid. J Orthop Res 33: 359-365, 2015.

36. Antonsson B: Bax and other pro-apoptotic Bcl-2 family 'killer-proteins' and their victim the mitochondrion. Cell Tissue Res 306: 347-361, 2001.

37. Fan TJ, Han LH, Cong RS and Liang J: Caspase family proteases and apoptosis. Acta Biochim Biophys Sin (Shanghai) 37: 719-727, 2005. 